\title{
QoS Oriented Opportunistic Routing protocol for Wireless Sensor Networks
}

\author{
Quentin Lampin*†, Dominique Barthel*, Isabelle Augé-Blum ${ }^{\dagger}$, and Fabrice Valois ${ }^{\dagger}$ \\ *Orange Labs R\&D, Meylan, France. Email: firstname.lastname@ orange.com \\ ${ }^{\dagger}$ Université de Lyon, INRIA, INSA-Lyon, Laboratoire CITI, F-69621. Email: firstname.lastname@insa-lyon.fr
}

\begin{abstract}
In the field, Wireless Sensor Networks face the harsh reality of radio physics, including asymmetric and long-range transient links. Classic routing schemes are hindered by these links, and therefore prune them from the network topology. In this paper, we investigate how to put these opportunistic wireless links to good use and actually provide higher quality of service. We propose QOR, short for QoS oriented Opportunistic Routing protocol for data collection in Wireless Sensor Networks. Unlike classic routing schemes, QOR takes advantage of opportunistic links to provide faster and more reliable transmissions.

Our contribution is threefold. First, we propose a joint routing structure and addressing scheme that allows to identify a limited set of nodes than can become opportunistic relayers between a source sensor and the sink. Second, we define an original cascaded acknowledgement mechanism that brings reliable acknowledgment and replication-free forwarding to the opportunistic communication scheme. Finally, our performance evaluation assesses that QOR efficiently uses opportunistic links to provide reliable and replication-free data delivery.
\end{abstract}

\section{INTRODUCTION}

As technologies for WSNs mature, the field of applications of these networks keeps expanding from wild-life habitat monitoring to automated data collection in smart cities [1]. Nowadays, such networks are subject to severe Quality of Service constraints, mainly delivery ratio, delay and, of course, lifetime. Due to the transient nature of wireless links, building a routing topology that meets such QoS requirements remains a key challenge. Thus, existing routing schemes have been adapted and optimized for WSNs and new routing protocols has been proposed [2], [3], [4], etc. Moreover, the necessity to connect sensor devices to the Internet led to the emergence of an IETF routing protocol, named RPL [5], that is optimized for data collection in wireless sensor networks.

However, classic routing schemes face a dilemma when addressing the two antagonistic QoS objectives: delivery ratio and delay. Indeed, to ensure a high delivery ratio, classic routing schemes use reliable and short range wireless links and prune long range, transient and asymmetric links. Unfortunately, those opportunistic links are the key to meet the low end-to-end delay requirement.

S. Biswas et al. [6] introduced the opportunistic routing scheme for wireless networks. Unlike in classical routing protocols, packets are broadcasted to all 1-hop neighbors. Upon receiving a packet, a node decides if the packet needs

This work has been partially funded by French Agence Nationale de la Recherche under contract VERSO ARESA2 2009-017. to be forwarded or discarded according to the evaluation of a progress estimator. This opportunistic scheme therefore divides the delivery and delay problem into two separate subproblems: "What is the set of forwarder candidates?" and "Which candidate should forward a given packet?" that can be solved separately. However, opportunistic routing protocols also introduce new issues that limit their adoption by both the scientific and industrial communities: packet replication, i.e. packets forwarded multiple times, and the lack of reliable acknowledgements, e.g. nodes having an asymmetrical link with the initiator of the transmission may decide to forward a packet while their acknowledgement cannot be received by it.

This paper introduces QOR, QoS oriented Opportunistic Routing protocol for data collection in WSNs. To optimize the use of opportunistic wireless links and to cope with replication issue, QOR builds a joint prefix-based addressing scheme and routing structure. To deal with asymmetric links, a "cascading" acknowledgement scheme is used. QOR's routing structure is a DODAG (Destination Oriented Directed Acyclic Graph) that offers multiple reliable paths between sensors and the sink to ensure high delivery ratios [7]. QOR's addressing scheme is based on hierarchical prefixes which allow nodes to compute the progress estimator and make the forwarding decision: the forwarder with the shortest prefix common with that of the initiator relays the packet. This guarantees a fast transit of the packets and therefore ensures low end-to-end delays. From this addressing scheme, QOR derives a framebased and collision-free acknowledgement scheme that serves two purposes: $i$ ) to reliably deliver the acknowledgement to the initiator using a multi-hop communication paradigm, ii) to avoid packet replication caused by multiple forwarders.

The rest of this paper is organized as follows: section II reviews existing opportunistic routing protocols and section III focuses on QOR's algorithm. Section IV highlights simulation results that assess the benefits of QOR. Finally, section V summarizes this article and unveils future work.

\section{RELATED WORK}

This section provides an overview of existing opportunistic routing protocols and a brief introduction to RPL. More details are given in [5].

ExOR [6], proposed by S. Biswas et al., is an opportunistic routing protocol for 802.11 wireless networks. In ExOR, initiators broadcast packets in batches to the 1-hop neighbors. Each packet includes an ordered list of candidate forwarders. 
The order of the candidates is derived from the ETX metric [8]. Upon reception, a candidate stores the packet if and only if its address is included in the candidates list. At the end of a batch, ExOR schedules the acknowledgements of transmissions according to the order of the candidates list. Acknowledgements, grouped in a "batch map", are sent back to the initiator by the candidate forwarders in reverse order of their respective priority. Batch maps are updated by each candidate, such that every received packet is acknowledged to the initiator. At the end of this procedure, candidates send the packets that have not been acknowledged by higher priority candidates: this reduces packet replication. Several optimizations of ExOR have been proposed. MORE [9] aims at reducing duplicated packets by using random linear combinations of packets and simple algebra to detect duplicated packets. Z. Zhong et al. [10] proposes an optimization of the candidate selection and introduces a prioritization mechanism based on a novel metric (EAX, expected any-path transmissions).

Recently, attempts to adapt the opportunistic routing scheme to wireless sensor networks have emerged. For example, J. Carnley et al. implemented TORP [11] (which behaves like ExOR) on TinyOS. To our understanding, TORP still requires a global knowledge of the network topology, which is too costly or unrealistic for large wireless sensor networks.

Because QOR finds a part of its inspiration in the IETF routing protocol for low power and lossy networks (RPL), we underline here the latter's key mechanisms. RPL [5] is an IPv6 routing protocol that builds a Destination Oriented Directed Acyclic Graph (DODAG) based on customizable objective functions (OF) and metrics [12]. RPL is designed to efficiently support data collection flows, i.e. convergecast traffic to a few sinks. The DODAG routing topology is built in a distributed manner and originates at the sinks. Sinks broadcast advertisement packets (named DODAG Information Objects, DIOs), that contain a scalar value (the Rank), and possibly metric descriptors and values. Nodes may join the DODAG and choose one or more of their advertising neighbors as potential next-hops for the convergecast traffic. Such neighbors are called parents and one of them, the one that is deemed the most worthy according to the OF, is called the preferred parent. Nodes that join the DODAG may further propagate DIOs with updated ranks and metrics to reflect their position in the structure.

\section{QOR}

This section describes the QOR protocol. Section III-A gives the big picture and the main ideas behind QOR while sections III-B to III-C provide an in-depth description of QOR algorithm.

\section{A. In a Nutshell}

- QOR is a novel opportunistic routing protocol for data collection in wireless sensor networks.

- QOR builds a stable, reliability-tuned DODAG routing structure that ensures a bidirectional connectivity between sensors and the sink.
- Each node is given its address and delegated an address subdomain by its parent in the DODAG. These address and subdomain are picked in the address domain of the parent.

- Nodes broadcast their data packets.

- Upon receiving a packet, a node compares the initiator's address to its subdomain. If it is in its subdomain, i.e. the node is an ancestor of the initiator, it participates in the relaying of the packet, otherwise it drops the packet.

- Ancestors acknowledge the transmission in a slotted frame in the descending order of their subdomain inclusion (enclosing subdomain first). Because of the addressing scheme, two ancestors cannot have the same subdomain size, therefore acknowledgements are collision-free.

- Ancestors listen to the slots that precede their own acknowledgement slot. If an ancestor hears an earlier acknowledgement, it drops the data packet, otherwise it becomes responsible for relaying the packet. In both cases, the ancestor transmits an acknowledgement.

- This process of transmitting up the ancestor line is repeated until the packet reaches the sink.

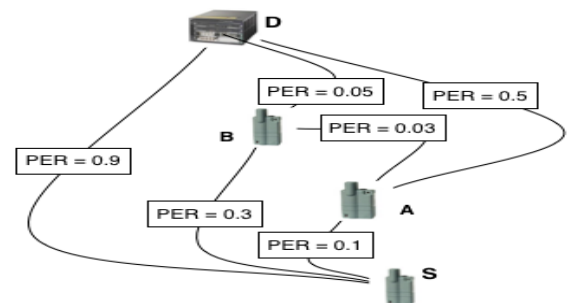

Fig. 1: Improving the delivery ratio, delay and energy consumption using QOR

To illustrate the interest of using an opportunistic routing protocol like QOR, let us consider fig 1. In such a scenario where $\mathbf{S}$ has a packet for $\mathbf{D}$, a classic routing protocol would select route S-A-B-D because it's the most reliable. Without link layer acknowledgement and retransmission, a total of 3 transmissions are needed for any successful delivery, and the expected success ratio is 0.83 . With link acknowledgement and unlimited retransmission, the expected number of transmissions for successful delivery is 3.19. With QOR, $\mathbf{S}$ delivers the packet to $\mathbf{D}$ in one transmission with probability $p=0.1$, in two transmissions with $p=0.67$ and in 3 hops with $p=0.11$. With QOR without retransmission, the expected number of transmissions for a successful delivery is therefore 2.01 and the expected success ratio is 0.88 . With link acknowledgement and unlimited retransmission, the expected number of transmissions for successful delivery is 2.27 .

\section{B. Construction of the Directed Acyclic Graph structure}

QOR focuses on building a reliable routing structure to ensure that all nodes obtain a reliable and bi-directional path to the sink. As in RPL, QOR builds a DODAG rooted at the sink. Nodes participating in the DODAG may have multiple parents. Each node of the DODAG sends advertisement messages 


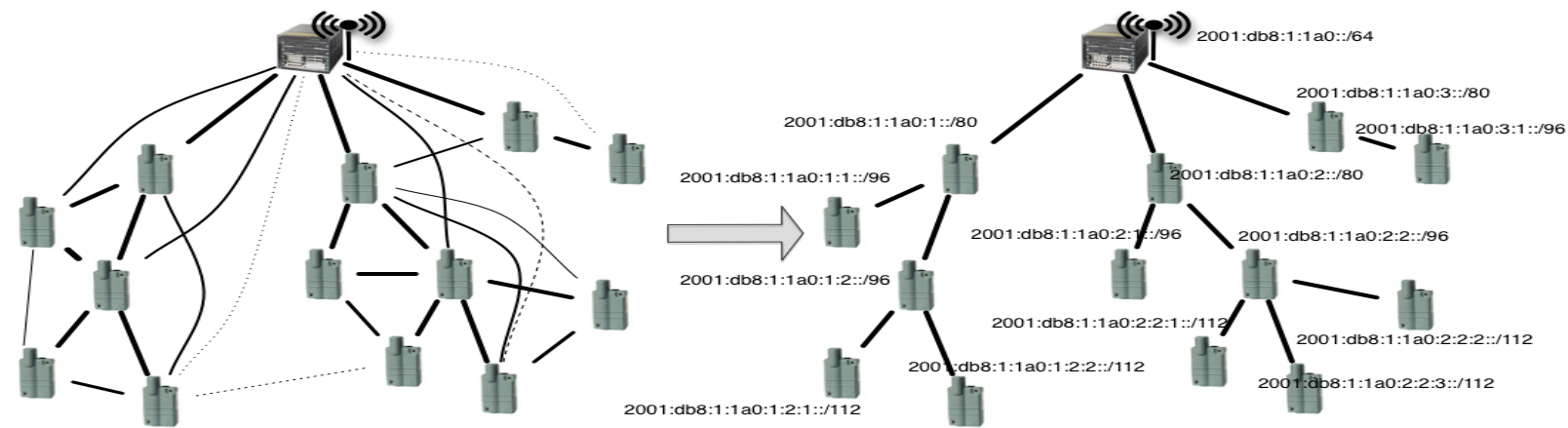

Fig. 2: QOR routing structure and addressing plan

(DIO) that include its rank and its metric. In QOR, the rank is defined as the depth of a given node, i.e. the number of hops in the structure between a given node and the sink.

1) Computation of the metric: The metric represents the quality of the path between a given node and the sink. We assume that both the Radio Signal Strength Indicator and its variability are sufficient to identify reliable and stable wireless links [13]. Thus, the metric is based on the evaluation of the RSSI of packets exchanged on the links that compose the path to the sink. More specifically, the node metric is a couple $\left(m_{n}, s_{n}\right)$ where $m_{n}$ is the worst average link RSSI of the path to the root and $s_{n}$ is the standard deviation of $m_{n}$.

2) Selection of the preferred parent: The initial choice of the preferred parent candidate is subject to the computation of a Quality SCore (QSC) that reflects the reliability of the advertised paths. QSC is used to order potential parents and is computed as follows: $Q S C_{c}=m_{c}-s_{c}$. The QSC is a biased estimator of the average RSSI of the weakest link of the path. Using QSC leads to selecting reliable and stable links over unreliable and/or unstable links for routing purpose.

Once QSCs have been computed for every potential parent, a node $N_{\text {in }}$ that wishes to join the DODAG sends a DODAG Information Solicitation (DIS) to the potential parent with the highest QSC. Upon receiving the DIS, the preferred parent candidate sends a Reply message back. The Reply message contains either an address and a subdomain or a notice of refusal in case it cannot accept the request (e.g. address space depleted, poor uplink quality, etc). In the latter case, $N_{\text {in }}$ retries with the potential parent with the second highest QSC and repeats this process until it joins the routing structure or runs out of potential parents.

3) Selection of the alternate parents: Nodes may elect alternate parents to prevent disconnections from the DODAG in case the preferred parent gets unreachable. The selection of the alternate parents follows the same mechanism as for the preferred parent but are subject to supplementary conditions. First, nodes may attach to an alternate parent only if the parent address is not included in its own subnet. Second, nodes may not attach to nodes whose QSC is significantly lower than the QSC of its preferred parent $p$, i.e. lower than $P Q S C=m_{p}-2 * s_{p}$, a Pessimistic QSC of the preferred parent.
4) Topology adaptation: A node in the DODAG may decide to update its parents due to a change in the topology or in the radio environment. Several cases can occur: $i$ ) a node may detach from an alternate parent in case its QSC score is too low, ii) it may change its preferred parent if an alternate node provides a more reliable route to the sink, iii) it may also look for adding new alternate parents. In any case, a topology change must meet the criteria described in Section III-B3. Furthermore, if a topology inconsistency is detected and requires a change of node address, the node sends an Update message to its children providing the new addressing. Last, if a node becomes disconnected from the sink, it sends a DIO with an infinite rank to its children so that they detach from it.

5) Path advertisement: After joining the DODAG, a node may advertise a path to the sink by sending DIOs. Those DIOs contain the address obtained from the preferred parent, the node's rank and the node metric $\left(m_{n}, s_{n}\right)$. The DIOs transmissions are controlled by a Trickle timer [14]. In Trickle, the timer period $I$ is re-evaluated over time according to a consistency criterion. When neither a topology inconsistency is detected nor a DIS is received for some time, the value of $I$ is doubled, until it reaches a maximum value $I_{\max }$. On the contrary, if a node receives a DIS or detects a topology inconsistency, it resets $I$ to its minimal value $I_{\min }$. This achieves a good trade-off between quick reaction to changes and low energy during stable phases. In QOR, $I_{\min }$ is a small value relative to the duty-cycle of the sensor.

\section{Addressing scheme}

Previously, we introduced the routing structure for the data collection. In this section, we present the prefix-based addressing scheme which has two complementary objectives: firstly, it allocates a collision-free address to each node, secondly, it allows detecting and using long range opportunistic wireless links up the ancestor chain by comparing the packet initiators' addresses to nodes' subdomains.

Upon joining the DODAG, a sensor gets an address and a subset of the address domain from its preferred parent. This subdomain of the global address domain is guaranteed to be unique and non overlapping: this ensures that every node gets a collision-free address. The subdomain is described by the 
subdomain prefix and the address range. Because of the strict inclusion relation of the address subdomains, the farther a node from the root, the smaller its subnet.

Fig. 2 provides a comprehensive example of how the addressing scheme is applied. It uses IPv6 notation conventions. At the root, the sink has an address 2001:db8:1:1a0::0 and a /64 subnet, with the left-most 64 bits equal to that of the sink. Nodes that join the DODAG at the first rank, i.e. one hop from the sink, get an address from the sink amongst $2001: \mathrm{db} 8: 1: 1 \mathrm{a} 0: \mathbf{1}:: 0,2001: \mathrm{db} 8: 1: 1 \mathrm{a} 0: \mathbf{2}:: 0$, $2001: \mathrm{db} 8: 1: 1: \mathrm{a} 0: \mathbf{f f f f}:: 0$ and a /80 subnet. Nodes that join at rank 2 get an address and a subnet from their preferred parents and so on. For example, the rightmost sensor of Fig.2 gets an address from $2001: \mathrm{db} 8: 1: 1 \mathrm{a} 0: 3:: 0$, i.e $2001: \mathrm{db} 8: 1: 1 \mathrm{a} 0: 3: 1:: 0$ and a /96 subnet.

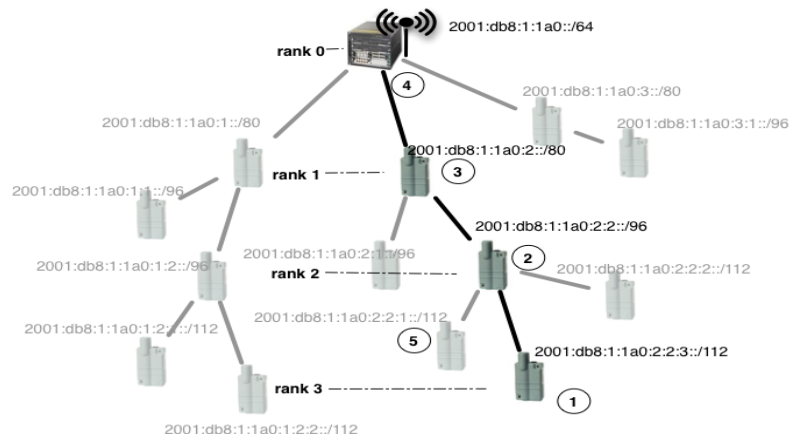

Fig. 3: QOR addressing scheme: address allocation, subdomain inclusion and potential forwarders.

1) Selection of the forwarder set: As shown in Fig. 3, in QOR, the set of potential forwarders for a given packet is determined by the packet initiator's address. A node is in the forwarder set if it is an ancestor of the packet initiator, i.e. the initiator's address is in the node's subdomain. Because nodes addresses are unique and address subdomains do not overlap, the forwarding candidates forms a linear subgraph of the DODAG, that has neither branches nor loops.

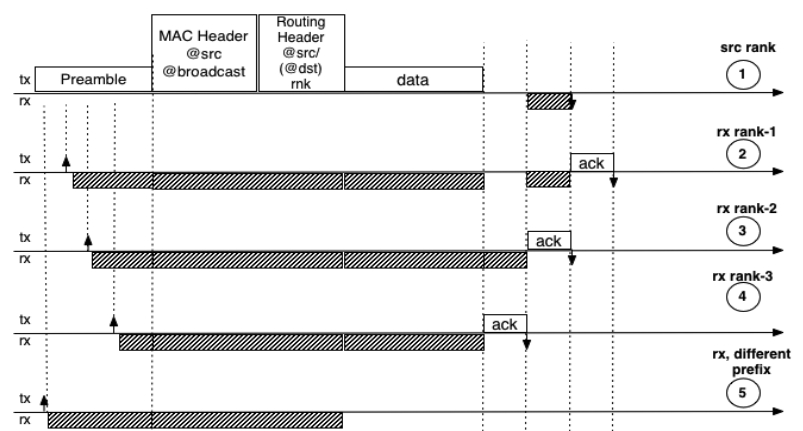

Fig. 4: QOR relies on a slotted acknowledgment frame which is demonstrated on a B-MAC-like MAC protocol, i.e. longpreamble, source initiated MAC protocol.

2) Acknowledgement scheme: The acknowledgement scheme has two objectives, $i$ ) it elects the ancestor closest to the root as the single forwarder of the data packet, $i i$ ) it reliably conveys the acknowledgement from the forwarder back to the packet initiator, potentially over multiple hops. To do so, QOR defines a slotted acknowledgement frame as shown in Fig. 4. The first slot is reserved for the sink, and the following slots are mapped to the packet initiator ancestors in the descending order of their subdomain size, i.e. nodes closer to the sink get the earlier slots of the frame. Because of the linearity of the ancestors subset and the strict inclusion relation between the ancestors subdomains, there is only one ancestor per slot. This guarantees collision-free acknowledgement slots.

QOR defines the sole forwarder as the first ancestor to send an acknowledgement in the frame, i.e. the closest to the sink. To reliably convey the acknowledgement back to the initiator, ancestors listen to the slots preceding their own. If an ancestor hears an acknowledgement, it discards the data packet (it will not forward it) and repeats in its own slot the acknowledgment heard. The acknowledgment is therefore conveyed safely to the initiator over multiple hops in a single acknowledgement frame.

3) Detailed example of the forwarder selection and acknowledgement schemes: Figs 3 and 4 illustrate the selection and acknowledgement schemes of QOR. In Fig. 3, node $\mathbf{1}$ sends a packet to the sink $\mathbf{4}$. Nodes $\mathbf{2}$ to $\mathbf{5}$ receive the packet. 5's subdomain does not include 1's address, so it is not an ancestor. It therefore discards the packet upon receiving the routing header. Other nodes receive the full packet and schedule their acknowledgements. 4 picks the first slot as it is the destination of the packet while $\mathbf{2}$ and $\mathbf{3}$ respectively send their acknowledgements in slots $\mathbf{3}$ and 2. Let's assume $\mathbf{1}$ and $\mathbf{2}$ do not receive the acknowledgement sent by $\mathbf{4}$ while $\mathbf{3}$ does. $\mathbf{3}$ retransmits the acknowledgement from $\mathbf{4}$, discards the packet and turns its radio off. Its acknowledgement is received by $\mathbf{1}$ and 2. Upon reception of the acknowledgement, the initiator, $\mathbf{1}$, turns its radio off. 2 finally retransmits the acknowledgement in the third slot, discards the packet and turns its radio off.

\section{Evaluation}

\section{A. Simulation Setup}

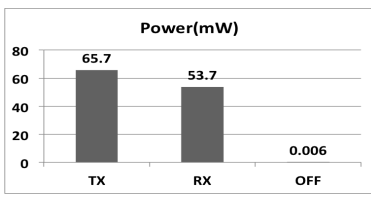

(a) radio model

\begin{tabular}{|c|c|}
\hline simulation time & $100000 \mathrm{~s}$ \\
\hline radio bitrate & $20 \mathrm{kbits} / \mathrm{s}$ \\
\hline TX power & $0 \mathrm{dBm}$ \\
\hline CS sensitivity & $-97 \mathrm{dBm}$ \\
\hline propagation & two ray ground \\
\hline noise & $\begin{array}{c}\text { AWGN } \mu=-115 \mathrm{dBm} \\
\sigma=1 \mathrm{~dB}\end{array}$ \\
\end{tabular}

(b) simulation parameters
Fig. 5: Simulation settings

The evaluation has been conducted on the WSNET network simulator [15] which provides an accurate modeling of interferences in wireless multihop networks. The simulations use an energy model based on the power consumption of the states and transitions of an actual $19.2 \mathrm{kbits} / \mathrm{s}$ commercial radio chip. The emission power and the carrier sense sensitivity have been set to $0 \mathrm{dBm}$ and $-110 \mathrm{dBm}$ respectively. We set the propagation 
model to the two ray ground model on an AWGN channel. The noise average power is set to $-115 \mathrm{dBm}$ while its standard deviation is set to $1 \mathrm{dBm}$.

This performance evaluation provides a comparison between QOR and a classic distance vector routing protocol that implements the RPL specifications that we call RPL in the rest of the paper, for the sake of simplicity. This implementation of RPL is tuned to provide high delivery ratios and therefore favors reliable and stable paths. To compare the two protocols, we have simulated them on two types of physical topologies: a set of grids of increasing density and population (49 to 225 sensors) and a random topology of 200 sensors. The grids topologies evaluate the protocols in dense scenarios, i.e. 30$100+$ sensors $/ \mathrm{km}^{2}$, while the random topology evaluates them in a sparse setup: $10-15$ sensors $/ \mathrm{km}^{2}$ whose characteristics match an existing sensor network.

In each scenario, a source node located at the border of the topology generates data traffic that is collected at the sink, located at the center of the topology. The period of the traffic ranges from $1 \mathrm{~min}$ to $3 \mathrm{mins}$ to illustrate various traffic congestions. With these setups, source nodes generate 500 to 1500 packets in each simulation.

In this performance evaluation, we measure the delivery ratio, the end-to-end delay, the energy consumption and we count replicated packets. The rationale is that we expect to illustrate a significant gain on the end-to-end delay and energy consumption while preserving a very high delivery ratio, hence proving that QOR uses opportunistic links to improve the delay and decrease the energy consumption.

\section{B. Delivery Ratio}

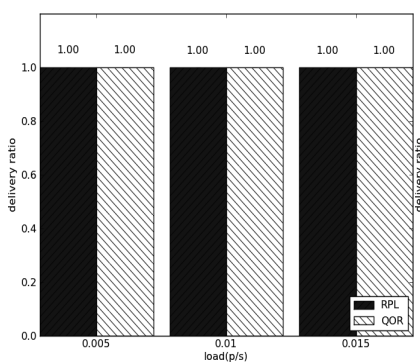

(a) 169 nodes grid topology
Fig. 6: Delivery ratio

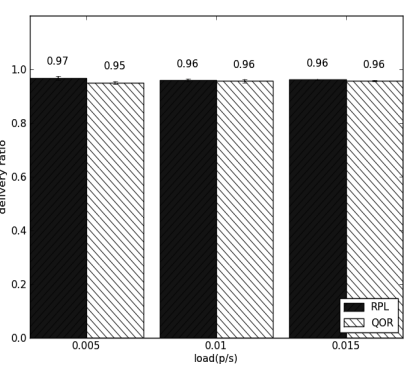

(b) Random topology
As expected, simulations show that the implementation of RPL performs very well in terms of delivery ratio with a minimum of $0.994 \%$ on the random topology. Interestingly, as illustrated on Fig. 6, QOR performs as well as its competitor, the difference being less than $0.2 \%$. However, this result is interesting because it assesses that QOR allows using opportunistic links in a reliable fashion.

\section{End to End delay}

While the delivery ratio is very similar for the two protocols, QOR clearly outperforms RPL for the end-to-end delay (figures 7 and 8). Fig. 7 illustrates that the use of opportunistic

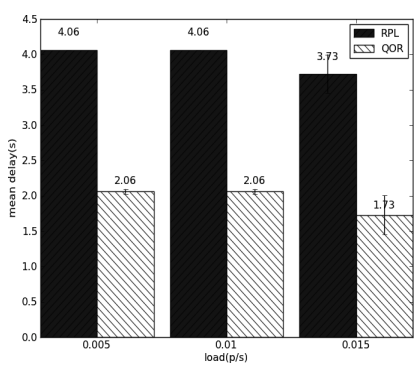

(a) 49 nodes

(c) 169 nodes

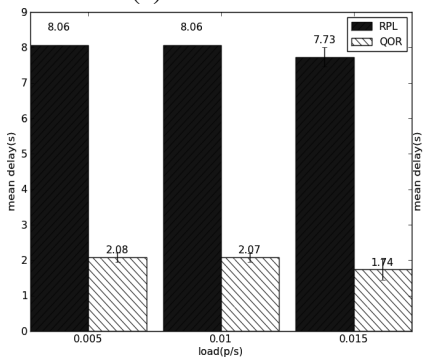

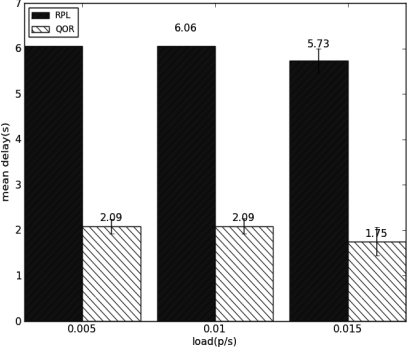

(b) 81 nodes

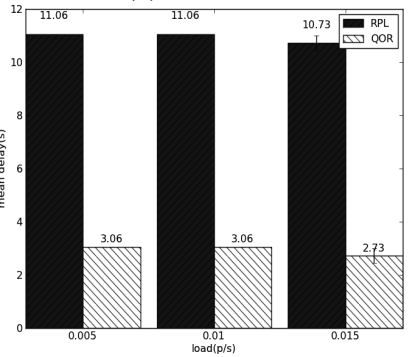

(d) 225 nodes
Fig. 7: Grids topology: End-to-End Delay.

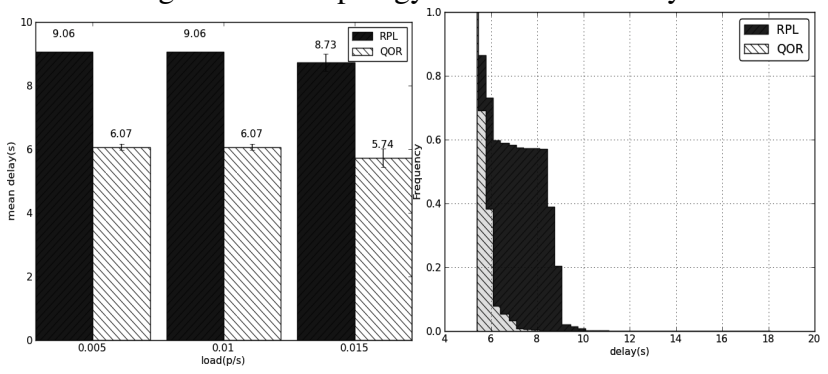

(a) Mean delay

(b) Inverse CDF of the delay

Fig. 8: Random topology: End-to-End Delay

links allows QOR to significantly decrease the end-to-end delay. In our simulations, QOR reduces the end-to-end delay by as much as a factor of four in the densest topology. As expected, the denser the topology, the higher the benefit: this is due to more long-range, opportunistic links becoming available for QOR to use.

As shown on Fig. 8, the same observation holds true on the sparse random topology. QOR indeed shows a solid $30 \%$ decrease in the end-to-end delay compared to RPL. Fig. $8 \mathrm{~b}$ illustrates another interesting property of QOR: the inverse Cumulative Distribution Function of the delay shows a tighter delay spread with QOR (5 to 8s) than with RPL (6s to $11 \mathrm{~s}$ ), which makes delivery times more predictable even though QOR uses unreliable and transient links.

\section{Energy Consumption}

A question that comes to mind with such results is the impact on the energy consumption of using opportunistic links. As shown in Section III-C2, multiple ancestors are involved in the routing of acknowledgements to the initiator, which comes at a price, and packet replication may also lead to a waste of nodes energy. 


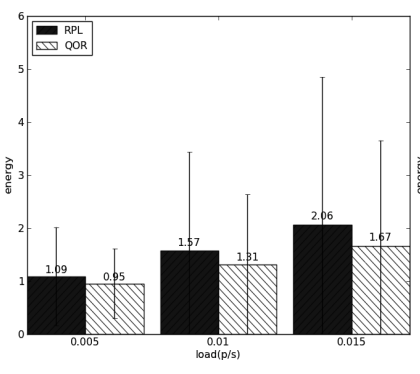

(a) 49 nodes

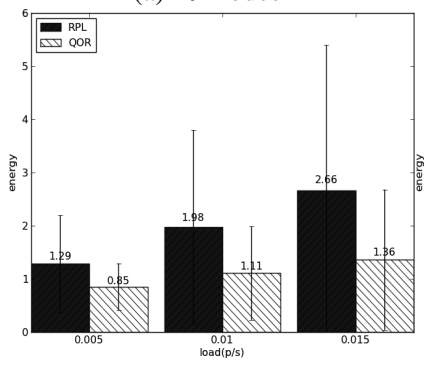

(c) 169 nodes

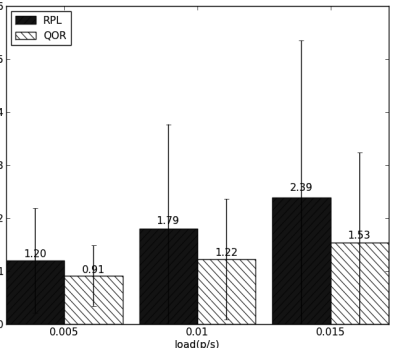

(b) 81 nodes

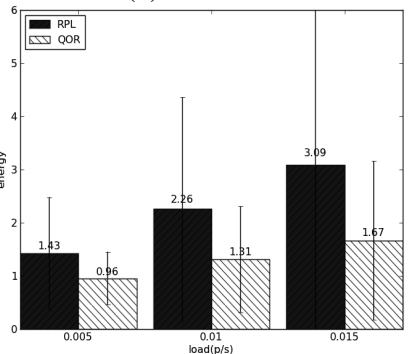

(d) 225 nodes
Fig. 9: Grid topologies: power consumption

Interestingly, Fig. 9 shows that, instead of increasing the energy consumption, QOR consumes as little as half the energy of RPL on dense topologies. Indeed, because it uses long range links, QOR reduces the total number of transmissions that are required to forward a packet from a source to the sink. As illustrated on Fig. 5a, the main source of energy dissipation stems from an active radio: $\sim 50 \mathrm{~mW}$ in RX/TX vs $\sim 5 \mu \mathrm{W}$ when the radio is off. By reducing the amount of transmissions required in the delivery of a data packet, QOR lowers the overall energy consumption. This also suggests that packet replication is rare: the highest ratio of replicated packets to total receptions that we observed is less than $0.8 \%$. Fig. 10

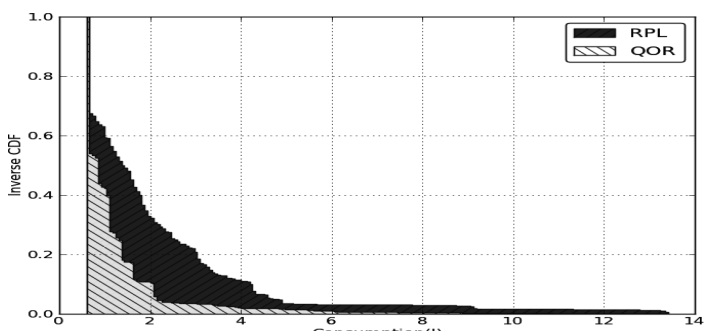

Fig. 10: Inverse CDF of the power consumption

sheds light on the distribution of the power consumption and underlines another interesting property of opportunistic routing protocols, especially true in the case of QOR. The CDF of the energy consumption shows a smaller range of values in the case of QOR. This means that the sensors lifetime will bear less variability with QOR than with RPL.

On the sparse random topology, QOR shows an average decrease in the power consumption of $4 \%$ when compared to RPL, which leads to the conclusion that QOR protocol overhead is low enough that its energy consumption is still lower than that of RPL while bringing about a significant
$(30 \%)$ decrease in the end-to-end delay.

\section{E. Results synthesis}

The performance evaluation highlights that QOR provides a reliable transmission scheme for data collection wireless sensor networks while using opportunistic links. Using such opportunistic links, QOR significantly improves the end-toend delay and power consumption while achieving delivery ratios similar to that of an implementation of RPL crafted for maximum reliability. Simulations show that QOR produces very few replicated packets and a low enough overhead to achieve significant improvements over a state of the art protocol on a wide range of network densities.

\section{CONCLUSION AND FUtURE WORK}

This paper introduces QOR, a QoS oriented Opportunistic Routing protocol that is designed for data collection in wireless sensor networks. QOR brings a practical way of efficiently and reliably using opportunistic wireless links. Using such links, QOR reduces the end-to-end delay and the energy consumption while offering high delivery ratios. QOR is therefore a good candidate to support traffic loads facing antagonistic constraints such as delivery and delay. QOR is best suited for dense networks but still performs well on sparse topologies.

Future works on QOR include studying the impact of the metric choice on the network performance as well as proposing an implementation of QOR on top of RPL, while still being interoperable. We also envision a test-bed experiment to confirm the simulation results.

\section{REFERENCES}

[1] M. Dohler, T. Watteyne, T. Winter, and D. Barthel, "Routing requirements for urban low-power and lossy networks," RFC 5548, 2009.

[2] I. Amadou, G. Chelius, and F. Valois, "Energy-efficient beacon-less protocol for wsn," in PIMRC, sept. 2011.

[3] H. Kalosha, A. Nayak, S. Ruhrup, and I. Stojmenovic, "Select-andprotest-based beaconless georouting with guaranteed delivery in wireless sensor networks," in INFOCOM, april 2008.

[4] N. Mitton, T. Razafindralambo, D. Simplot-Ryl, and I. Stojmenovic, "Hector is an energy efficient tree-based optimized routing protocol for wireless networks," in $M S N$, dec. 2008.

[5] T. Winter and P. Thubert, "RPL: IPv6 Routing Protocol for Low Power and Lossy Networks," RFC 6550, 2011.

[6] S. Biswas and R. Morris, "Exor: opportunistic multi-hop routing for wireless networks," in SIGCOMM, 2005.

[7] Q. Lampin, D. Barthel, and F. Valois, "Efficient route redundancy in dag-based wireless sensor networks," in WCNC, april 2010.

[8] D. S. J. De Couto, D. Aguayo, J. Bicket, and R. Morris, "A highthroughput path metric for multi-hop wireless routing," in MobiCom, 2003.

[9] S. Chachulski, M. Jennings, S. Katti, and D. Katabi, "Trading structure for randomness in wireless opportunistic routing," in SIGCOMM, 2007.

[10] S. N. Z. Zhong, J. Wang and G.-H. Lu, "On selection of candidates for opportunistic anypath forwarding," SIGMOBILE, October 2006.

[11] J. Carnley, B. Sun, and S. Makki, "Torp: Tinyos opportunistic routing protocol for wireless sensor networks," in CCNC, jan. 2011.

[12] T. Winter and P. Thubert, "Routing Metrics used for Path Calculation in Low Power and Lossy Networks," RFC 6551, 2011.

[13] K. Srinivasan and P. Levis, "Rssi is under appreciated," in EmNets, 2006.

[14] P. Lewis, T. Clausen, J. Hui, O. Gnawali, and J. Ko, "The trickle algorithm," RFC 6206, 2009.

[15] E. B. Hamida, G. Chelius, and J.-M. Gorce, "Impact of the physical layer modeling on the accuracy and scalability of wireless network simulation," Simulation, 2009. 